\title{
Man muss lange leben, um ein Mensch zu werden
}

(Antoine de Saint-Exupéry)

Vor nicht allzu langer Zeit wurde eine 80jährige Chirurgin angeklagt, die gewagt hatte, in ihrem hohen Alter noch das Skalpell zu benutzen und zu operieren. Angeklagt wurde sie aber nicht deswegen, sondern weil sie ein unübliches Operationsverfahren angewandt hatte. Dabei tauchte - nicht ganz überraschend - die Frage auf, wie lange eine Ärztin überhaupt praktizieren darf bzw. wann man mit seiner ärztlichen Tätigkeit aufhören sollte.

Viele freipraktizierende Ärzte hören im Gegensatz zu ihren angestellten Kollegen nicht von einem Tag auf den andern mit ihrer Arbeit auf, sondern reduzieren ihr Pensum allmählich. Oft behandeln sie noch während einiger Jahre über das Pensionierungsalter hinaus völlig unbeanstandet langjährige Patienten mit chronischen Leiden. Diese sind ihnen dafür auch sehr dankbar. Ich denke, die meisten Hausärzte und Spezialisten wissen genau, was sie können, und sind in der Lage, ihre Fähigkeiten auch nach 65 noch richtig einzuschätzen. Meine Erfahrungen mit verschiedenen älteren Kollegen bestätigen diese Annahme ausnahmslos. Dies lässt sich sicher auch damit erklären, dass Ärzte während der gesamten Zeit ihrer beruflichen Tätigkeit immer wieder die Qualität ihrer Arbeit selbstkritisch hinterfragen müssen.

\section{Die Gelassenheit ist eine anmutige Form des Selbstbewusstseins}

(Marie von Ebner-Eschenbach)

Im Gegensatz zu den freipraktizierenden Ärzten werden unsere Kollegen in den Kliniken in der Regel von einem Tag auf den andern pensioniert. Mit Ausnahme von ganz wenigen Universitätsordinarien müssen Chefärzte zwingend, spätestens an ihrem 65. Geburtstag, in den Ruhestand treten, dies unabhängig davon, wie gut sie noch arbeiten können und wie gerne sie noch weiterarbeiten würden. Doch um wen handelt es sich bei diesen «aus Altersgründen Entlassenen»? Es sind Ärzte verschiedener Fachrichtungen, die meist eine mindestens 30jährige konstante Berufserfahrung haben. Auch wenn vielleicht gewisse manuelle Fähigkeiten und manchmal die «Effizienz» ihrer Arbeit etwas nachgelassen haben, so sind sie doch noch normal leistungsfähig. Sie stehen wahrscheinlich nachts nicht mehr so leicht auf und erholen sich nach einer durchgearbeiteten Nacht auch nicht mehr so schnell, aber sie machen dies durch ihre enorme Erfahrung mehr als wett. Neben diesem enormen Wissens- und Erfahrungsschatz haben sie meistens auch etwas mehr Geduld. Alles in allem ein Potential, das nun plötzlich nicht mehr genutzt wird und daher verkümmert. Aber genau dieser Wissensschatz und diese Erfahrung wären für die Ausbildung unserer jungen Kollegen von enormem Wert! Ihre Gelassenheit und ihr Einfühlungsvermögen in unserem hektischen Alltag ein willkommenes Gegengewicht.

\section{Mit dem Alter nimmt die Urteilskraft zu und das Genie ab}

(Immanuel Kant)

Genau dies ist der Vorteil der älteren, erfahrenen Ärzte gegenüber der jungen Generation. Es wäre eine gute Investition, wenn sich die Kliniken einen pensionierten Chefarzt leisten würden, der noch zu einem kleinen Prozentsatz angestellt wäre, um die Weiterbildung der jungen Ärzte zu unterstützen. Dies würde es ermöglichen, unseren jungen Kollegen eine besser strukturierte Weiterbildung zu bieten, da sie sich gezielter und kontinuierlicher der Lehre widmen könnten. Noch idealer wären flexiblere Pensionierungsvarianten, die es ermöglichen würden, erfahrene Ärzte über das Pensionsalter hinaus als Consultant in der Aus- und Weiterbildung oder auch in anderen klinischen Bereichen zu beschäftigen. Natürlich könnten dazu auch ehemals freipraktizierende Ärzte eingesetzt werden.

Wir wollen keinesfalls die Weiterbildung aus dem klinischen Alltag entfernen. Es bleibt wichtig, dass die Kaderärzte weiterhin im Alltag lehren. Aber durch die Anstellung von speziell dafür vorgesehenen «Lehrmeistern» wäre es möglich, die Weiterbildung zu strukturieren und $\mathrm{zu}$ vertiefen und damit für unsere jungen Kollegen auf einem hohen Niveau auch eine Kontinuität sicherzustellen. Dies dank den älteren Kollegen mit ihrer grossen Berufs- und Lebenserfahrung!

Dr. med. Brigitte Muff, Mitglied des Zentralvorstandes der FMH 